\title{
Primeros registros de incremento en los niveles plasmáticos de glucosa en individuos adultos de Pleurodema thaul (Lesson, 1826) (Anura: Leptodactylidae) sometidos a estrés por frío
}

\section{First records of increased plasma glucose levels in adults of Pleurodema thaul (Lesson, 1826) (Anura: Leptodactylidae) subjected to cold stress.}

\author{
Pabla Hernández $z^{1,2 *}$, Helen Díaz-Páez $Z^{1}$ \& Eduardo Tarifeño-Silva². \\ ${ }^{1}$ Departamento de Ciencias Básicas, Campus Los Ángeles, Universidad de Concepción. Casilla 341, Los Ángeles, Chile. \\ ${ }^{2}$ Departamento de Zoología, Campus Concepción, Universidad de Concepción. Casilla Casilla 160-C, Concepción, Chile. \\ *E-mail: pahernandezc@udec.cl
}

\begin{abstract}
RESUMEN
El presente estudio analizó las variaciones en la concentración plasmática de glucosa en el anfibio, Pleurodema thaul sometido a estrés térmico por frío. Para ello, se midieron las concentraciones plasmáticas de este metabolito utilizando un glucómetro Accu-Chek Active y un kit comercial. Los resultados muestran significativos incrementos de glucosa en los individuos sometidos al estrés térmico por frío, por lo que se identifica a la glucosa como elemento anticongelante en esta especie.
\end{abstract}

Palabras clave: Anfibios, crioprotector, estrés térmico, glucosa.

\begin{abstract}
This study analyzed the synthesis of glucose in Pleurodema thaul exposed to cold thermal stress as an antifreezing molecule. The plasma glucose concentrations were measured using a glucose meter Accu-Chek Active and a commercial kit. The results showed significant increase of plasma glucose in individuals under the cold thermal stress, indicating that glucose have a function as antifreeze mechanism in this species.
\end{abstract}

KEYWORDs: Amphibians, cryoprotectant, thermal stress, glucose.

\section{INTRODUCCIÓN}

Es conocido que la temperatura juega una función clave en la regulación de las funciones fisiológicas y conductuales de los animales ectotermos (Anguiletta, 2009), sin embargo se ha determinado que éstos poseen una capacidad termorreguladora muy limitada (Navas 1997). Anfibios y reptiles, obtienen desde el medio externo la energía calórica requerida para incrementar su temperatura corporal y así poder ejecutar sus funciones metabólicas de rutina (Sanabria et al. 2007). Esto convierte a la temperatura en un factor ambiental selectivo de primer orden ya que influye en la supervivencia, crecimiento y dispersión de estos individuos (Angilletta 2009). Para poder responder a las presiones del entorno durante los periodos fríos, los animales deben disponer de diversas estrategias de comportamiento, fisiológicas y bioquímicas que les permitan sobrevivir
(Pinder et al. 1992; Storey \& Storey 2001). En anfibios, una de estas estrategias es la hibernación, la cual se logra mediante: i) inmersión en el agua, ii) permanencia en tierra en un invernáculo térmicamente aislado, donde algunos organismos se resguardan de las duras condiciones climáticas al esconderse bajo la hojarasca o la nieve, y iii) permanencia en tierra con tolerancia al congelamiento, que se asocia a mecanismos adaptativos que impiden que los fluidos extracelulares alcancen el punto de congelación (Pinder et al. 1992; Storey \& Storey 2001; Hill et al. 2006; Weels 2007; Tattersall \& Ultsch 2008).

Para evitar el daño celular y tisular frente a las lesiones físicas producidas por la formación de cristales de hielo en el proceso de congelación-descongelación, los individuos producen diversas moléculas crioprotectoras, las que permiten mantener y reactivar las funciones vitales después de días o semanas de congelación continua (Layne \& Lee 1995). 
Los crioprotectores constituyen una amplia gama de compuestos orgánicos (Storey \& Storey 1986; Layne \& Lee 1995; Storey \& Storey 1996; 2001;; Costanzo \& Lee 2013) que se incorporan a los líquidos corporales (Hill et al. 2006) tanto intra como extracelulares, lo que determina el incremento de la concentración de solutos en ambos compartimentos y por lo tanto un aumento de la presión osmótica de dichos compartimentos. De acuerdo a las propiedades coligativas, al aumentar la cantidad de soluto en los líquidos extracelulares disminuye el punto de congelamiento, limitando la formación del hielo en este compartimento. Mientras que el incremento de la concentración de los solutos en los líquidos intracelulares limita la cantidad de agua que debe salir de las células lográndose un equilibrio osmótico con los líquidos extracelulares que están concentrados (Storey \& Storey 1996; Hill et al. 2006).

Entre los crioprotectores reconocidos destacan las proteínas anticongelantes, cuyo efecto es la disminución del punto de congelación de los líquidos corporales (Storey \& Storey 1996; Steiner et al. 2000; Hill et al. 2006). Este tipo de proteínas han sido descritas en varios tipos de organismos, como bacterias, plantas, peces marinos polares, en algunos insectos y arañas (Storey \& Storey 1996; Sinclair et al. 2003; Antony et al. 2016) y se ha determinado que su funcionamiento básico no impide la formación y crecimiento de los cristales de hielo, pero lo limitan a tamaños manejables que no comprometen las funciones vitales de la célula (Hill et al. 2006).

Junto a esto se ha determinado que moléculas tales como glucosa, el glicerol y la urea actúan disminuyendo el punto de congelación de los líquidos corporales de acuerdo a los principios coligativos; es decir, disminuyen el punto de congelación al incrementar la concentración total de solutos en los líquidos y no por medio de propiedades químicas específicas. El mecanismo de producción de glucosa extracelular cómo agente crioprotector se activa cuando el anfibio se enfrenta a condiciones de frío extremo y los individuos son capaces de producir cantidades masivas de glucosa a partir de reservas de glucógeno, en forma súbita frente a la formación de hielo en sus cuerpos (Hill et al. 2006, do Amaral et al. 2013). Este tipo de crioprotectores han sido reportados como los más recurrentes en anfibios viviendo en altas latitudes del Hemisferio Norte (Storey \& Storey 1996; Hillman et al. 2009; Costanzo \& Lee 2013) estudios que destacan la glucosa como molécula crioprotectora en al menos seis especies de anfibios anuros: Rana sylvatica, Lithobates catesbeianus, Pseudacris triseriata, Acris crepitans, Pseudacris crucifer y Rhinella schneideri (Costanzo \& Lee 1993; Layne et al. 1996; Irwin et al. 1999; Edwards et.al. 2000; Steiner et al. 2000; Jenkins \& Swanson 2005; Costanzo \& Lee 2013; do Amaral et al. 2013; Rosendale et al. 2014). Recientemente, Sanabria et al. (2015) reportaron la respuesta fisiológica de sobreenfriamiento en Rhinella spinulosa en Argentina, indicando como posible mecanismo utilizado por la especie, el incremento en los niveles de glucosa plasmática, lo que no fue confirmado experimentalmente.

El anfibio, Pleurodema thaul, llamada comúnmente "sapito de cuatro ojos", corresponde a una especie de anuro nativa del sur de Sudamérica, descrita para Chile y Argentina. En Chile, es uno de los anfibios más abundantes (Díaz-Páez \& Ortiz 2001) debido a que ha logrado adaptarse a distintos tipos de hábitats, presentando una amplia distribución latitudinal, delimitada entre Carrera Pinto, Región de Atacama (27 $06^{\circ} \mathrm{S}, 6^{\circ} 53^{\prime} \mathrm{O}$ ) en el norte (Correa et al. 2007), hasta Aysén ( $45^{\circ} 30^{\prime} \mathrm{S}, 70^{\circ} 20^{\prime} \mathrm{O}$ ) en el sur (Cei 1962; Duellman \& Veloso 1977; Victoriano et al. 1995; Díaz-Páez \& Ortiz 2001; Iturra-Cid 2010), y desde la costa hasta $3.125 \mathrm{~m}$ sobre el nivel del mar (Correa et al. 2010). Esta especie es capaz de vivir en ambientes térmicos tanto de bajas temperaturas como de altas temperaturas (Sommaruga 2001). En este contexto, destaca la mención de Iturra-Cid et al. (2014) quienes reconocen la capacidad de $P$. thaul para hibernar.

En base a los antecedentes anteriores, el presente estudio busca analizar que posibles mecanismos de tolerancia al congelamiento dispone el anfibio $P$. thaul, dado que esta especie soporta en Chile ambientes climáticos extremos, lo que podría explicar su amplia distribución en Chile y Argentina.

\section{MATERIALES Y METODOS}

Se colectaron 20 ejemplares del anfibio $P$. thaul (permiso del Servicio Agrícola Ganadero Resolución N 9411/2014), durante el periodo de verano - otoño del año 2015 en la localidad de Antuco, sector Los Pangues, Región del Biobío, Chile (37²3'38"S; 71²6'16”O) (FIG. 1). Esta área fue seleccionada por presentar periodos invernales con temperaturas bajo cero grado (Luebert \& Pliscoff 2006).

Las capturas fueron realizadas manualmente, según lo propuesto por (Donnelly et al. 1994) utilizando el "Protocolo para el control de enfermedades infecciosas de Anfibios durante estudios de campo" (Lobos et al. 2012). Los individuos colectados fueron transportados en cajas acondicionadas al Laboratorio de Ecofisiología de Herpetozoos del Campus Los Ángeles, de la Universidad de Concepción. Durante el cautiverio y estados experimentales fueron mantenidos en terrarios grupales $(20 \times 35 \times 15 \mathrm{~cm})$ y fueron alimentados "ad libitum" tres veces por semana con una dieta compuesta de gusanos de tebos, larvas de tenebrios y adultos de mosca del vinagre. Para la identificación individual se utilizó el reconocimiento fotográfico (Bradfield 2004; Kenyon et. al. 2009; 2010), mediante el patrón dorsal de manchas y colores de los individuos.

Para el desarrollo de los experimentos, la muestra fue 


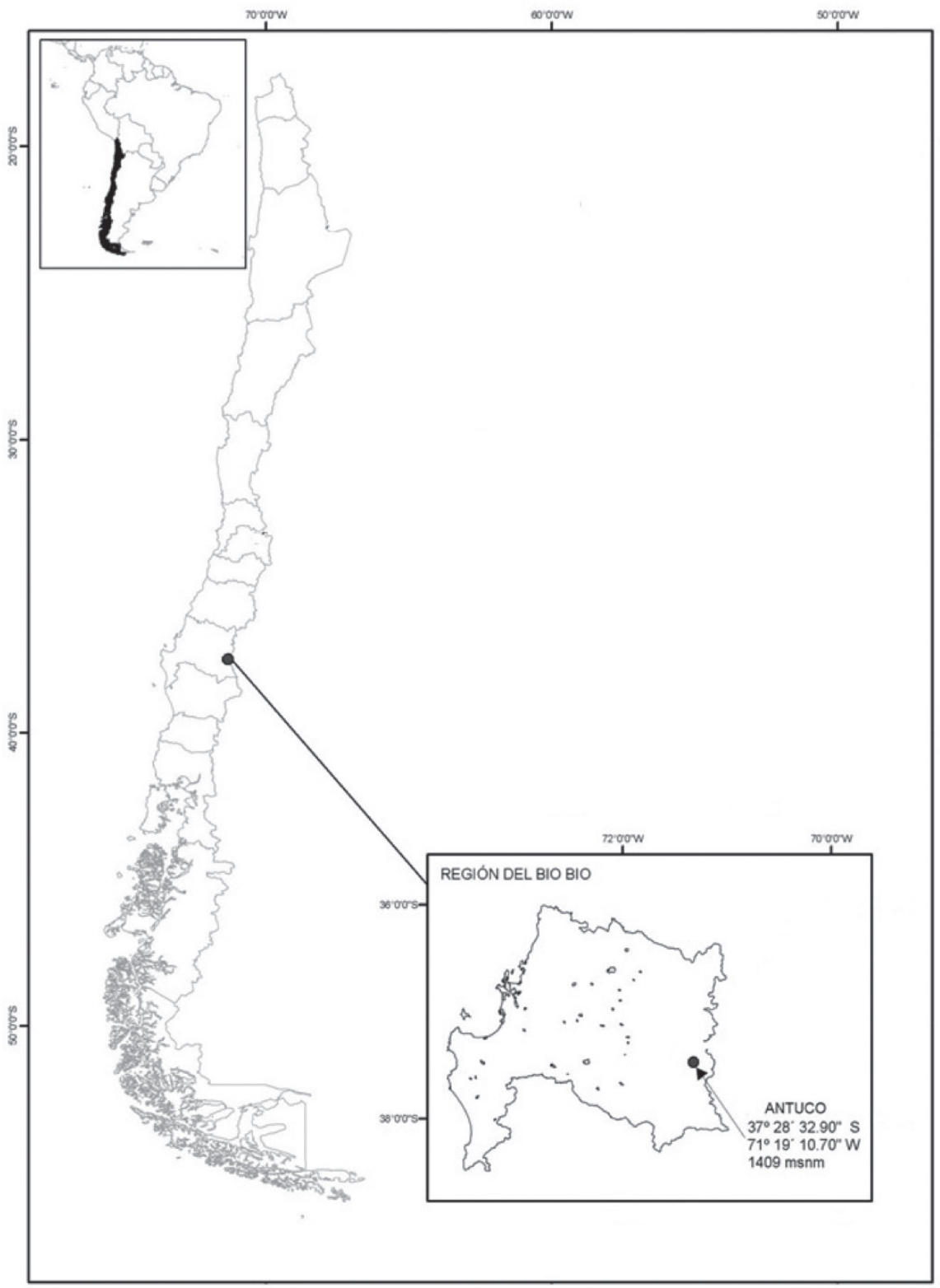

FIgURA 1: Ubicación geográfica del área de captura de los individuos de P. thaul. / Geographical location area of capture individuals of P. thaul.

separada en grupos de tres y cuatro individuos. Dos grupos de $\mathrm{n}=4$ fueron aclimatados a $25^{\circ} \mathrm{C}$ y humedad relativa del $60 \%$, con fotoperíodo de 14 horas de luz y 10 horas de oscuridad (simulando las condiciones ambientales promedio de primavera-verano) y dos grupos de $n=3$ fueron aclimatados a $5^{\circ} \mathrm{C}$, humedad relativa del $60 \%$ y fotoperíodo de 8 horas de luz y 16 horas de oscuridad (simulando las condiciones ambientales promedio de otoño-invierno). Finalmente, dos grupos de $n=3$ fueron mantenidos en condiciones ambientales normales (no controladas), para lo cual los terrarios se dejaron en un espacio abierto que simula las condiciones naturales de su hábitat, donde la temperatura, humedad y fotoperíodo corresponden a las fluctuaciones diarias normales en la ciudad de Los Ángeles, Región del Biobío, Chile. Todos los individuos asignados a cada tratamiento fueron escogidos de forma aleatoria.

Previo a cada experimento, se registró la temperatura corporal experimental desde el dorso del animal mediante un termómetro infrarrojo (Fluke 62 mini IR thermometer, $0,1^{\circ} \mathrm{C}$ de precisión). Se estableció como temperatura corporal inicial a la temperatura registrada al inicio del experimento y como temperatura corporal final a la temperatura registrada al finalizar la experimentación.

Después de cada experimento, contempló un periodo de una semana donde los anfibios se mantuvieron sin nuevas experimentaciones, para permitir su recuperación y retornar 
a sus condiciones fisiológicas normales (Storey \& Storey 1986; Layne et al. 1996). En promedio, la experimentación duró 4 meses, en los que se realizaron 2 eventos de experimentación mensual (Iturra-Cid 2010) (Fig. 2).

Para efectuar el protocolo de exposición al estrés térmico por frío, cada individuo fue puesto dentro de una caja plástica provista de respiración y papel absorbente húmedo, la que fue trasladada al interior de un sistema de refrigeración que se mantuvo a una temperatura constante de aproximadamente de $0^{\circ} \mathrm{C}$, simulando las temperaturas ambientales que se pueden encontrar durante la época invernal en el lugar de colecta (Luebert \& Pliscoff 2006). La temperatura del sistema experimental fue controlada con un termotester (Eosun EM Digital Multimeter 18 High Performance). Los animales permanecieron bajo estas condiciones durante cuatro horas, según los sugerido por Pinder et al. (1992). Para evitar el deceso de los animales, se revisó el estado del animal cada hora durante el proceso.

Para determinar los niveles de glucosa en la sangre, se realizó una punción con jeringa de insulina de 50 unidades y capacidad $0,5 \mathrm{ml}$ en la vena ventral del individuo, extrayendo una gota de sangre (menor al $0,1 \%$ de su peso corporal) (Allender et al. 2008; Tapley et al. 2011) la que fue inoculada en la cinta de lectura del glucómetro Accu-Chek Active. Esta metodología fue corroborada enzimáticamente usando un kit comercial (Spinreact). Para realizar este último proceso, las muestras de sangre fueron centrifugadas a $7000 \mathrm{rpm}$ durante 10 a 15 minutos. Se utilizaron 10 ul del plasma sobrenadante para realizar la reacción enzimática, la que fue incubada a $\mathrm{T}^{\circ}$ ambiente por 20 minutos y fuera del contacto con la luz, llevándola finalmente a espectrofotometría a $505 \mathrm{~nm}$ (Espectrofotómetro UV-VIS Genesys ${ }^{\mathrm{TM}} 10$, THERMO, precisión 1,0nm). Las medidas fueron registradas al inicio del protocolo de estrés por frío para cada individuo, denominándose nivel de glucosa inicial (Glui) y al finalizar éste, medida que corresponde al nivel de glucosa final (Gluf) una vez transcurridas 4 horas de estrés térmico. Las concentraciones de glucosa fueron expresadas en $\mathrm{mg} / \mathrm{dL}$.

\section{RESULTADOS}

Concentraciones de Glucosa plasmática medidas CON EL método enzimático SpinReact y Glucómetro Accu-CheK Active.

Para determinar la correspondencia entre las concentraciones plasmáticas de glucosa obtenidas mediante la utilización del glucómetro Accu-Chek Active y el método enzimático con el kit comercial Spinreact, se utilizó el coeficiente de correlación de Spearman, encontrándose una alta correlación entre ambos instrumentos de medición cuando se compararon los Glui $\left(\mathrm{r}_{\mathrm{s}=} 0,892 ; \mathrm{P}<0,001\right)$, Gluf $\left(\mathrm{r}_{\mathrm{s}=}\right.$

Diseño experimental

\begin{tabular}{|c|c|c|c|}
\hline \multirow{4}{*}{ Mes 1} & Semana 1 & $\begin{array}{c}\text { Aclimatados a } 25^{\circ} \mathrm{C} \\
\text { Aclimatados a } 5^{\circ} \mathrm{C} \\
\text { Mantenidos a } \mathrm{T}^{\circ} \text { ambiente }\end{array}$ & $\begin{array}{c}\text { Aplicación del protocolo de } \\
\text { estrés térmico por frío. }\end{array}$ \\
\hline & Semana 2 & $\begin{array}{c}\text { Aclimatados a } 25^{\circ} \mathrm{C} \\
\text { Aclimatados a } 5^{\circ} \mathrm{C} \\
\text { Mantenidos a } \mathrm{T}^{\circ} \text { ambiente }\end{array}$ & $\begin{array}{c}\text { Recuperación de las condiciones } \\
\text { fisiológicas normales }\end{array}$ \\
\hline & Semana 3 & $\begin{array}{c}\text { Aclimatados a } 25^{\circ} \mathrm{C} \\
\text { Aclimatados a } 5^{\circ} \mathrm{C} \\
\text { Mantenidos a } \mathrm{T}^{\circ} \text { ambiente }\end{array}$ & $\begin{array}{l}\text { Aplicación del protocolo de } \\
\text { estrés térmico por frío. }\end{array}$ \\
\hline & Semana 4 & $\begin{array}{c}\text { Aclimatados a } 25^{\circ} \mathrm{C} \\
\text { Aclimatados a } 5^{\circ} \mathrm{C} \\
\text { Mantenidos a } \mathrm{T}^{\circ} \text { ambiente }\end{array}$ & $\begin{array}{c}\text { Recuperación de las condiciones } \\
\text { fisiológicas normales }\end{array}$ \\
\hline
\end{tabular}

FiguRa 2: Diseño experimental para el estudio de estrés térmico por frío en.P. thaul. / Experimental design for the study of thermal stress by cold in $P$. thaul. 
0,966; $\mathrm{P}<0,001)$ y las medidas en su conjunto (Glut) ( $\mathrm{r}_{\mathrm{s}}$ 0,936; $\mathrm{P}<0,001)$. Además, se corroboró la ausencia de diferencias significativas para las mediciones de glucosa plasmática entre ambas metodologías $\left(Z_{\text {(inicial) }}=-5,291 ; P\right.$ $\left.>0,05, Z_{\text {(final) }=}=-1,968 ; \mathrm{P}>0,05 \mathrm{y} \mathrm{Z}{ }_{\text {(total) }=}=-2,431 ; \mathrm{P}>0,05\right)$, confirmando que los datos obtenidos con ambos métodos no presentaron diferencias significativas (Fig. 3). Esto resultó de suma importancia porque avaló la utilización del glucómetro como método que puede ser utilizado en terreno y que a su vez resultó menos invasivo para los individuos, ya que utiliza un menor volumen sanguíneo.

VARIACIONES DE LOS NIVELES PLASMÁtICOS DE GLUCOSA.

Los niveles de glucosa plasmática inicial (Glui) y final (Gluf) mostraron diferencias significativas entre los tratamientos de aclimatación $\left(5^{\circ} \mathrm{C}, 25^{\circ} \mathrm{C}\right.$ y $\mathrm{T}^{\circ}$ ambiente) (TABLA 1), de manera que los animales sometidos a menores temperaturas $\left(5^{\circ} \mathrm{C}\right)$ presentan niveles menores de Glui $(12,82 \pm 6,59)$ y Gluf $(14,51 \pm 4,82)$ que aquellos sometidos a una temperatura de aclimatación mayor $\left(25^{\circ} \mathrm{C}\right.$ ) (Glui:

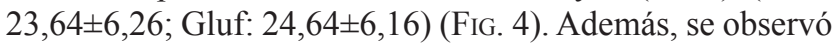
la ausencia de diferencias significativas en los valores de las concentraciones plasmáticas de glucosa Glui y Gluf para aquellos individuos de control que no fueron sometidos al protocolo de estrés por frío (Tabla 1).

Por otra parte, al aplicar previamente el protocolo de estrés por frío, se determinó la presencia de diferencias significativas en los niveles de glucosa plasmáticas Glui y Gluf para cada uno de los tres tratamientos de aclimatación (Tabla 1). Los resultados muestran que los individuos sometidos a la temperatura menor de aclimatación $\left(5^{\circ} \mathrm{C}\right)$ son lo que presentaron menores rangos para la glucosa plasmática (Glui: 7,37 $\pm 3,70$; Gluf: 17,72 $\pm 6,14$ ), mientras que aquellos que fueron sometidos a una mayor temperatura de aclimatación $\left(25^{\circ} \mathrm{C}\right)$ presentaron valores de glucosa

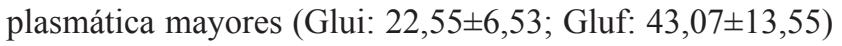
(Fig. 5). Cabe destacar que cuando los animales fueron sometidos al protocolo de estrés por frío, los niveles de glucosa final aumentaron en casi $20 \mathrm{mg} / \mathrm{dL}$, tanto en el tratamiento de aclimatación de $25^{\circ} \mathrm{C}$ (Gluf: $43,07 \pm 13,55$ ) como en el tratamiento de temperatura ambiente (Gluf: $46,91 \pm 15,84$ ) (Tabla 1 ).

Finalmente, se encontró una relación directa inversa entre el descenso de temperatura y el aumento de glucosa plasmática, en todos los tratamientos de aclimatación. Es así como para el grupo mantenido a $25^{\circ} \mathrm{C}$ el descenso de temperatura al que fueron sometidos los animales provoca un aumento significativo de glucosa plasmática $\left(\mathrm{Z}_{\text {Temp-Gluc= }}\right.$ $-3,884 ; \mathrm{P}<0,001)$, lo mismo que para el tratamiento de $5^{\circ} \mathrm{C}$ $\left(Z_{\text {Temp-Gluc }=}-3,662 ; \mathrm{P}<0,001\right)$ y a $\mathrm{T}^{\circ}$ ambiente $\left(\mathrm{Z}_{\text {Temp-Gluc }=}-4\right.$, 309; P $<0,001)$.

Adicionalmente, los resultados mostraron una correlación negativa entre los niveles de glucosa plasmática y la temperatura de aclimatación, la cual es mayor en los individuos aclimatados a $25^{\circ} \mathrm{C}$ y a $\mathrm{T}^{\circ}$ ambiente; es decir, los niveles de glucosa plasmática aumentaron más en estos grupos cuando fueron sometidos a estrés por frío $\left(0^{\circ} \mathrm{C}\right)$. En el grupo aclimatado a $5^{\circ} \mathrm{C}$, también se encontró una correlación negativa significativa; sin embargo, esta fue menor (Tabla 1).

Tabla 1: Niveles de glucosa plasmática inicial (Glui) y final (Gluf) en ejemplares de $P$. thaul sometidos a tratamientos de temperaturas de $5^{\circ} \mathrm{C}, 25^{\circ} \mathrm{C}$ y ambiente. Los valores son entregados como media \pm DS (desviación estándar). Análisis de test de Wilcoxon para las diferencias dentro de cada tratamiento son representados con valor de (Z). Análisis de test de Kruskal-Wallis para comparar las variaciones entre grupos de tratamiento son representados como $\mathrm{X}^{2}$ (NS: no significativo, $* \mathrm{P}<0,05 * * \mathrm{P}<0,005 * * * \mathrm{P}<0,001$ ). / Plasma glucose levels in specimens of $P$. thaul subjected to different temperature treatments. Data for variations initial glucose (glui) and final (Gluf) grouped by treatment $\left(5^{\circ} \mathrm{C}, 25^{\circ} \mathrm{C}\right.$ and $\mathrm{T}^{\circ}$ environment) are analyzed. Values are given as mean $\pm \mathrm{SD}$ (standard deviation). Wilcoxon test analysis for the differences within each treatment are represented with $(\mathrm{Z})$ value. Kruskal-Wallis test analysis to compare the variations between treatment groups are represented as $\mathrm{X}^{2}$ (NS: not significant, $* \mathrm{P}<0.05 * * \mathrm{P}<0.005 * * * \mathrm{P}<0.001$ ).

\begin{tabular}{|c|c|c|c|c|c|c|}
\hline & & & $5^{\circ} \mathrm{C}$ & $25^{\circ} \mathrm{C}$ & $\mathbf{T}^{\circ}$ ambiente & $\mathbf{X}^{2}$ \\
\hline \multirow{3}{*}{ Sin someter a estrés por frío } & & Glui & $12,82 \pm 6,59$ & $23,64 \pm 6,26$ & $31,58 \pm 11,74$ & $26,883 * * *$ \\
\hline & & Gluf & $14,51 \pm 4,82$ & $24,64 \pm 6,16$ & $29,19 \pm 8,57$ & $26,600 * * *$ \\
\hline & $\mathbf{Z}$ & & $-1,363^{\mathrm{NS}}$ & $-0,086^{\mathrm{NS}}$ & $-0,408^{\mathrm{NS}}$ & \\
\hline \multirow{3}{*}{ Sometido a estrés por frío } & & Glui & $7,37 \pm 3,70$ & $22,55 \pm 6,53$ & $26,42 \pm 8,23$ & $34,968 * * *$ \\
\hline & & Gluf & $17,72 \pm 6,14$ & $43,07 \pm 13,55$ & $46,91 \pm 15,84$ & $31,830 * * *$ \\
\hline & $\mathbf{Z}$ & & $-3,724 * * *$ & $-4,458 * * *$ & $-3,296 * * *$ & \\
\hline
\end{tabular}


Gayana 81(2), 2017
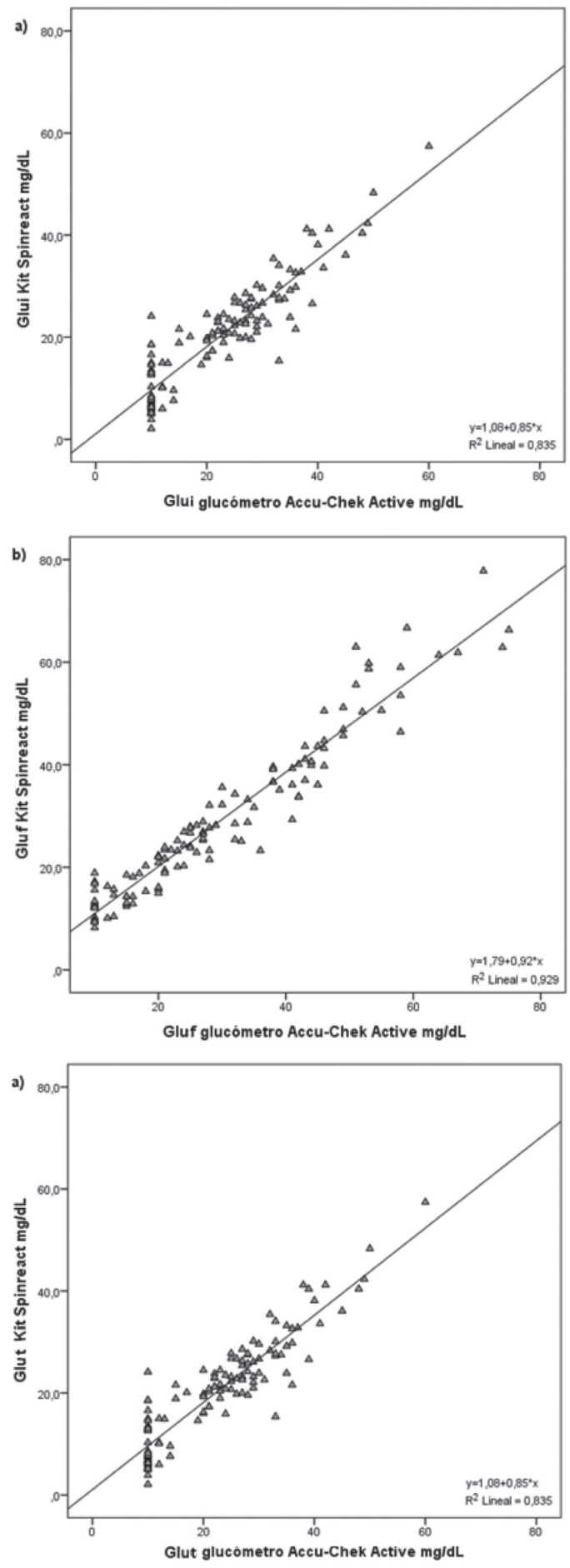

Figura 3: A) Correlación entre los niveles de glucosa plasmática (mg/dL) inicial (Glui)en todos los tratamientos, mediciones efectuadas con el glucómetro Accu-Check Active confrontadas con las mediciones realizadas con el método enzimático kit Spinreact; в) Correlación entre los niveles de glucosa plasmática (mg/dL) final (Gluf) en todos los tratamientos, medida con el glucómetro Accu-Check Active y el método enzimático con el kit Spinreact; c) Correlación entre los niveles de glucosa plasmática (mg/dL) total (inicial y final) (Glut) en todos los tratamientos, medidos con el glucómetro Accu-Check Active y el método enzimático con el kit Spinreact. / A) Correlation between plasma glucose levels (mg/dL) initial (Glui) in all treatments, measured with Accu-Check Active confronted with measurements made with enzymatic method, Spinreact kit. B) Correlation between plasma glucose levels (mg/dL) final (Gluf) in all treatments, measured with AccuCheck Active glucometer and the enzymatic method with Spinreact kit. c) Correlation between total plasma glucose levels (mg/dL) (initial and final) (Glut) in all treatments, measured with Accu-Check Active glucometer and the enzymatic method with Spinreact kit. 


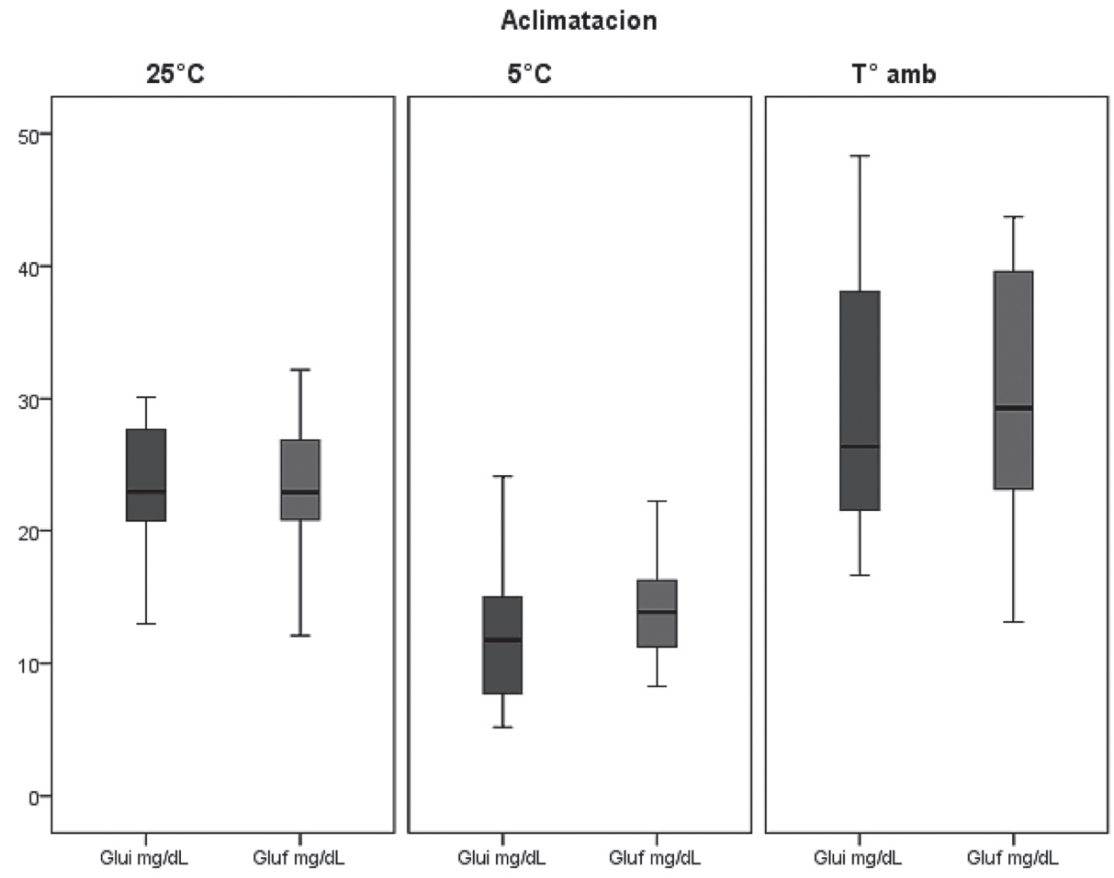

FIgURA 4: Niveles de glucosa plasmática (mg/dL) inicial (Glui) y final (Gluf), en individuos de $P$. thaul sometidos a los tres tratamientos de aclimatación, que no fueron expuestos al protocolo de estrés por frío. / Plasma glucose levels (mg / dL) Initial (Glui) and final (Gluf) in individuals of $P$. thaul under the three acclimation treatments that were not exposed to cold stress protocol.

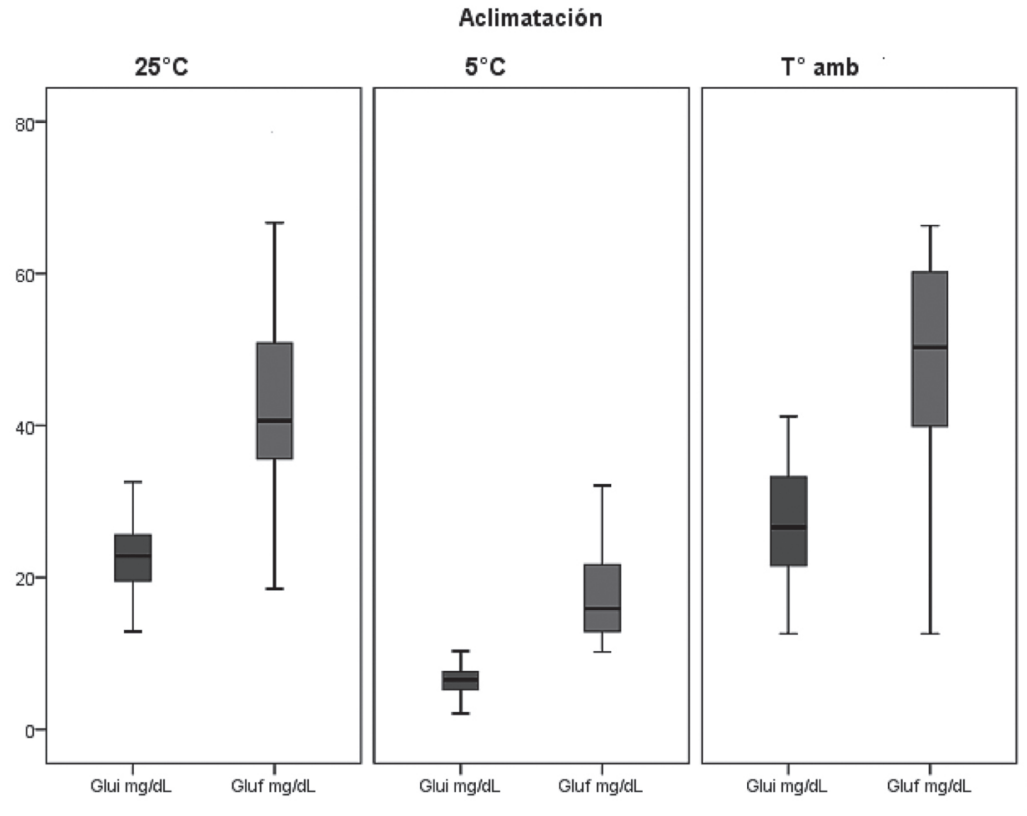

Figura 5: Niveles de glucosa plasmática (mg/dL) inicial (Glui) y final (Gluf) en individuos de $P$. thaul sometido a los tres tratamientos de aclimatación y expuestos al protocolo de estrés por frío. / Plasma glucose levels (mg / dL) Initial (Glui) and final (Gluf) in individuals of $P$. thaul under the three acclimation treatments that were exposed to cold stress prot. 


\section{DISCUSIÓN}

Los cambios fisiológicos de anfibios anuros sometidos a situaciones extremas de temperatura es una temática que ha sido tratada desde distintas perspectivas como la termorregulación, metabolismo, temperaturas corporales de campo, hibernación, tolerancia al congelamiento, entre otras, existiendo múltiples estudios al respecto (Brattstrom 1963; Eckert et al. 1991; Angilletta et al. 2002; Sanabria et al. 2003; 2007; Logares \& Úbeda 2006; Vidal \& Labra 2008; Angilletta, 2009; Iturra-Cid et al. 2014). En lo que concierne al uso de mecanismos de crioprotección, la mayor parte de la literatura se concentra en trabajos realizados en especies de ambientes extremos del Hemisferio Norte, donde destacan los trabajos que abordan los mecanismos de tolerancia al congelamiento en Rana sylvatica, Pseudacris crucifer, Pseudacris triseriata e Hyla versicolor (Storey \& Storey 1996; Wells 2007; Costanzo \& Lee 2013). Sin embargo, en el Hemisferio Sur este tópico ha sido poco estudiado (Felton et al. 2009), aun cuando se han realizado algunas menciones que indican que es posible que estos mecanismos estén presentes en algunas especies como un tipo de adaptación al frío extremo (Steiner et al. 2000; Sanabria et al. 2015).

Pese a la amplia información que ha indicado la existencia de tolerancia a la congelación en diversos taxa animales (Storey \& Storey 1986; 1996; Sinclair et al. 2003; Antony et al. 2016), los mecanismos que permiten a los anfibios tolerar condiciones de congelamiento no se han analizado en profundidad.

En Chile, los estudios de fisiología de adaptaciones térmica en anuros han indagado aspectos tales como los límites térmicos de tolerancia, temperaturas de selección de hábitat, temperaturas críticas máximas y mínimas, tasas de calentamiento y enfriamiento en las especies de Calyptocephalella gayi, Pleurodema thaul, P. bufoninum, Batrachyla taeniata y Rhinella spinulosa (Benavides 2003; Lambrinos \& Kleier 2003; Iturra.Cid 2010; Navas et al. 2010; Nova 2010; Iturra-Cid et al. 2014), pero no existen antecedentes sobre aspectos de tolerancia al congelamiento y crioprotección. Sin embargo, resulta destacable la reciente información de probables mecanismos de crioprotección en $R$. spinulosa (Sanabria et.al. 2015), especie que también se encuentra presente en territorio chileno, lo que abre la posibilidad de que estos mecanismos también se expresen en otras especies que habitan lugares de condiciones climáticas extremas en Chile.

En este sentido, cabe destacar que las condiciones climáticas a lo largo de Chile son muy variables considerando el amplio rango latitudinal de casi $40^{\circ}$ pero también la alta pendiente entre cordillera y nivel del mar haciendo del país un ambiente altamente heterogéneo con diferentes regímenes térmicos en latitud y en altura. En particular, la población de $P$. thaul de Antuco, ha logrado utilizar la glucosa plasmática para enfrentar las condiciones invernales que presenta esta localidad, las que pueden alcanzar temperaturas bajo los $0^{\circ} \mathrm{C}$ (Anguilletta 2009; Sanabria et al. 2015).

En el presente estudio se mostró que los niveles plasmáticos de glucosa en $P$. thaul aumentan con el descenso de la temperatura, por lo que se indica que podría funcionar como crioprotector. Sin embargo, falta por analizar los mecanismos a nivel celular y tisular para verificar esta hipótesis. La utilización de glucosa para protección tisular frente al descenso de la temperatura parece un mecanismo bastante factible, basado en la fácil movilización que tiene la glucosa en el organismo, ya que deriva del glucógeno hepático sin necesidad de una reacción anabólica de síntesis (Storey \& Storey 1986; Costanzo et al. 1993; Jenkins \& Swanson 2005), por lo que se deduce que esta ruta metabólica es mucho más rápida y eficiente energéticamente para los individuos.

Cabe destacar que dentro de los crioprotectores que se han descrito para anfibios anuros, la glucosa ha sido reconocida como el mecanismo más recurrente entre estos organismos (Layne \& Lee 1987; 1995; Costanzo et al. 1992; 1993; Pinder et al. 1992; Costanzo \& Lee 1994; 2005; Irwin et al. 1999; Edwards et al. 2000; Steiner et al. 2000; Jenkins \& Swanson 2005; Larson et.al., 2014; Rosendale et.al.,2014), presentando una relación proporcional inversa; es decir, los niveles de glucosa incrementan a medida que la temperatura ambiental, y por ende la temperatura corporal, descienden. Estos antecedentes son concordantes con los resultados encontrados en el presente estudio para $P$. thaul, dado que, frente a la exposición al frío, la población estudiada presentó un aumento en la síntesis de glucosa, no importando la temperatura de aclimatación a la cual se hubiese sometido el grupo de estudio.

La tolerancia a la congelación sin duda ha evolucionado desde un conjunto de capacidades fisiológicas y bioquímicas preexistentes que se encuentran ampliamente presente en los anfibios (Eckert et al. 1991; Storey \& Storey 1996). Estudios realizados para $R$. sylvatica demostraron que la producción de glucosa se estimula sólo cuando estas ranas comienzan a congelarse (Storey \& Storey 1986; Costanzo et al. 1993;Rosendale et.al.,2014), lo que es similar con los cambios en los niveles de glucosa encontrados para $P$. thaul, ya que los cambios se apreciaron solo en los individuos sometidos al protocolo de estrés por frío, mientras que los individuos controles no sometidos a este protocolo mantuvieron los niveles de glucosa plasmática con variaciones no significativas.

Al analizar los resultados obtenidos en el presente estudio en los grupos por separado se encontró que existe una correspondencia entre el descenso de temperatura y el aumento de glucosa plasmática, la que es mayor en los individuos aclimatados a $25^{\circ} \mathrm{C}$ y aquellos mantenidos a temperatura ambiente, mientras que los individuos aclimatado a $5^{\circ} \mathrm{C}$ presentaron una menor alza en los niveles 
de glucosa plasmática; es decir, los niveles de glucosa plasmática no aumentaron radicalmente al someter a los individuos a estrés por frío dado que estos estaban ya acondicionados al estrés por frío. Esta diferencia, junto con corroborar la síntesis de glucosa en condiciones de bajas temperaturas, se debería a que los individuos permanecieron durante mucho tiempo en aclimatación (más de un mes) a $5^{\circ} \mathrm{C}$ y esta condición es considerada una temperatura crítica mínima (Iturra-Cid 2010). En este sentido sólo este grupo mostró un alza significativa de glucosa durante las primeras semanas de aclimatación, luego de lo cual los niveles de glucosa plasmática disminuyeron, ya que se estima, según Storey \& Storey (2001), que la glucosa debió ser almacenada en órganos como el cerebro y corazón, donde la glucosa evitaría el congelamiento a nivel tisular de órganos vitales del individuo (Pinder et al., 1992; Storey \& Storey, 2001). Esto sería concordante con lo descrito en otros anuros por Storey \& Storey (1986), ya que la síntesis de glucosa como crioprotector en los anuros deriva del catabolismo de glucógeno hepático y las reservas que se hayan establecido durante el verano y principios del otoño mediante la alimentación.

Se ha determinado que tras la descongelación los organismos son extremadamente hiperglucémicos, superando el umbral renal de reabsorción de glucosa, por lo que esta se excreta en la orina abundantemente, especialmente durante las primeras horas después de la descongelación (Layne et al. 1996). Sin embargo, este recurso no se pierde completamente, porque la glucosa se reabsorbe de manera efectiva desde la vejiga (Costanzo \& Lee 1997) y luego se acumula en forma de glucógeno hepático, quedando disponible para ser utilizado en el próximo evento de congelación (Storey \& Storey 1986). Sin embargo, el proceso de eliminación de la glucosa es mucho más lento que el de la síntesis inducida por la congelación y puede tomar más de una semana a $4^{\circ} \mathrm{C}$ para que la glucosa vuelva a los niveles exhibidos antes del congelamiento (Storey \& Storey 1986), de aquí la importancia de respetar el protocolo experimental que contempla la mantención de una semana, como mínimo, sin experimentación para permitir que los individuos logren retornar a sus condiciones fisiológicas normales.

Finalmente, no se puede descartar que las variaciones encontradas en el presente estudio puedan verse influidas por otros factores como el nivel de glucógeno hepático alcanzado antes de comenzar el descenso térmico (Kessi et.al.,1996; Rosendale et.al., 2014), lo que en condiciones ambientales normales ocurriría durante los meses de verano-otoño. Otro factor que no se analizó en este estudio fue el sexo de los individuos, debido a la escasa cantidad de hembras capturadas y/o actividad reproductiva, ya que cuando los individuos se encuentran activamente reproductivos (momento en el que es más fácil capturarlos), la mayor parte de la energía se deriva a la producción de huevos (principalmente en las hembras) (Iturra-Cid 2010) que para el caso de $P$. thaul tiene un ciclo casi continuo con actividad gametogénica durante el otoño, el invierno, y la primavera (Díaz-Páez \& Ortiz 2001), lo que claramente influiría negativamente en la síntesis de crioprotectores. Sin embargo, esto debe ser analizado en futuros estudios.

\section{AGRADECIMIENTOS}

Al Servicio Agrícola y Ganadero (SAG, permiso de colecta $\mathrm{N}^{\circ}$ : 9411/2014) y a Beca Conicyt 2013-2014, $\mathrm{N}^{\circ}$ folio beca 22130895. Está investigación fue patrocinada por el Proyecto VRID 213.413.010-1.0 de la Universidad de Concepción.

\section{REFERENCIAS}

Allender, M., Fry, M. 2008. Amphibian Hematology. Veterinary Clinics of North America: Exotic Animal Practice 11: 463480.

Angilletta, M., Niewiarowskib, P., Navas, C. 2002. The evolution of thermal physiology in ectotherms. Journal of Thermal Biology 27: 249-268.

Angilletta, M. 2009. Thermal adaptation: a theoretical and empirical synthesis. Oxford University Press, USA. 289 pp.

Antony, S., Buddle, C., Sinclair, B. 2016. Thermal biology and immersion tolerance of the Beringian pseudoscorpion Wyochernes asiaticus. Polar Biology 39: 1351-1355.

BenAvides, G. 2003. Biología térmica de Bufo spinulosus: efecto de la temperatura sobre el desarrollo larval, una comparación intraespecífica. Tesis de Doctorado, Universidad de Chile. Santiago. Chile. 108 pp.

Bradfield, K. 2004. Photographic identification of individual Archey's Frogs, Leiopelma archeyi, from natural markings. DOC Science Internal Series. Department of Conservation, Wellington. N.Z. 36 pp.

Brattstrom, B. 1963. A preliminary review of the thermal requirements of amphibians. Ecology 44: 238-255.

CEI, J.M. 1962. Batracios de Chile. Ediciones de la Universidad de Chile, Santiago de Chile. 128 pp. + cviii.

Costanzo, J., Lee, R. 1993. Cryoprotectant production capacity of the freeze-tolerant wood frog, Rana sylvatica. Canadian Journal of Zoology 71: 71-75.

Costanzo, J., Lee, R. 1994. Biophysical and Physiological Responses Promoting Freeze Tolerance in Vertebrates. International Union of Physiological Sciences/American Physiological Society 9: 252-256.

Costanzo, J., Lee, R. 1997. Frogs resorb glucose from urinary bladder. Nature 389: 343-344.

Costanzo, J., LeE, R. 2005. Cryoprotection by urea in a terrestrially hibernating frog. The Journal of Experimental Biology 208: 4079-4089.

Costanzo, J., Lee, R. 2013. Avoidance and tolerance of freezing in ectothermic vertebrates. The Journal of Experimental Biology 216: 1961-1967. 
Costanzo, J., Wright, M., Lee, R. 1992. Freeze tolerance as an overwintering adaptation in Copes' grey treefrog (Hyla chrysoscelis). Copeia 1992: 565-569.

Costanzo, J., Lee, R., Lortz, P. 1993. Physiological responses of freeze-tolerant and intolerant frogs: clues to evolution of anuran freeze tolerance. American Journal of Physiology 265: 721-725.

Correa, C., Sallaberry, M., GonzÁlez, B., Soto, E., Méndez, M. 2007. Notes on geographic distribution: Amphibia, Anura, Leiuperidae, Pleurodema thaul: Latitudinal and altitudinal distribution extension in Chile. Check List 3: 267-270.

Correa, C., Riveros, E., Lobos, G., Velásquez, N. 2010. Amphibia, Anura, Leiuperidae, Pleurodema thaul (Lesson, 1827): Altitudinal distribution extension and new records from its northern range. Check List 6: 10-12.

Díaz- PÁez, H., Ortiz, J.C. 2001. The reproductive cycle of Pleurodema thaul (Anura, Leptodactylidae) in central Chile. Amphibia-Reptilia 22: 431-445.

Do Amaral, M., Lee, R., Costanzo, J. 2013. Enzymatic Regulation of Glycogenolysis in a Subarctic Population of the Wood Frog: Implications for Extreme Freeze Tolerance. PLoS ONE 8(11): e79169. doi:10.1371/journal.pone.0079169.

Donnelly, M., Guyer, C., Juterbock, J., Alford, R. 1994. Techniques for marking amphibians. En: Heyer, W., Donnelly, M., McDiarmid, R., Hayek, L., Foster, M. (Eds). Measuring and monitoring biological diversity. Standard methods for amphibians . 277-284. Smithsonian Institution Press, Washington \& London.

Duellman W., Veloso A. 1977. Phylogeny of Pleurodema (Anura: Leptodactylidae): a biogeographic model. Occasional Papers of the Museum of Natural History, University of Kansas 64: 1-46.

Eckert R., Randall, D., Augustine, G. 1991. Fisiología animal: mecanismos y adaptaciones. Interamericana. McGrawHill, España. 436 pp.

Edwards, J., Koster, K., Swanson, D. 2000. Time course for cryoprotectant synthesis in the freeze-tolerant chorus frog, Pseudacris triseriata. Comparative Biochemistry and Physiology A 125: 367-375.

Felton, A., Fischer, J., Lindenmayer, D., Montague-Drake, R., Lowe, A., Saunders, D., Felton, A., Steffen, W., Munro, N., Youngentob, K., Gillen, J., Gibbons, P., Bruzgul, J., Fazey, I., Bond, S., Elliott, C., Macdonald, B., Porfirio, L., Westgate, M., Worthy, M. 2009. Climate change, conservation and management: an assessment of the peerreviewed scientific journal literature. Biodiversity and Conservation 18: 2243-2253.

Hill, R., Wise, G., Anderson, M. 2006. Fisiología Animal. Editorial Médica Panamericana. España. 1020 pp.

Hillman, S., Withers, P., Drewes, R., Hillyard, S. 2009. Ecological and environmental physiology of amphibians. Oxford University Press, USA. 469 pp.

Irwin, J., Costanzo, J., Lee, R. 1999. Terrestrial hibernation in the northern cricket frog, Acris crepitans. Canadian Journal of Zoology 77: 1240-1246.

ITURRA-CID, M. 2010. Variación geográfica de la ecología térmica de Pleurodema thaul (Amphibia: Leiuperidae) en Chile: adaptación local, potencial evolutivo y consecuencias en un escenario de cambio climático. Tesis de Magíster. Universidad de Concepción. Concepción. Chile. 57 pp.
Iturra-Cid, M., Vidal, M., Labra, A., Ortiz, J.C. 2014. Winter Thermal Ecology Of Pleurodema thaul (Amphibia: Leptodactylidae). Gayana 78: 25-30.

JenKIns, J., SWANSON, D. 2005. Liver glycogen, glucose mobilization and freezing survival in chorus frogs, Pseudacris triseriata. Journal of Thermal Biology 30: 485-494.

Kenyon N., Phillott A., Alford R. 2009. Evaluation of photographic identification method (PIM) as a tool to identify Litoria genimaculata (Anura: Hylidae). Herpetological Conservation and Biology 4: 403-410.

Kenyon N., Phillott A., Alford R. 2010.Temporal variation in dorsal patterns of juvenile green- eyed tree frogs, Litoria genimaculata (Anura: Hylidae). Herpetological Conservation and Biology 5: 126-131.

Kessi, E., Guixe, V., Preller, A., Ureta, T. 1996. Glycogen synthesis in amphibian oocytes: evidence for an indirect pathway. Biochemical Journal 315: 455-460.

Lambrinos, J., Kleier, C. 2003. Thermoregulation of juvenile Andean toads (Bufo spinulosus) at $4300 \mathrm{~m}$. Journal of Thermal Biology 28: 15-19.

Larson, D., Middle, L., Vu, H., Zhang, W., Serianni, A., Duman, J., BARNES, B. 2014. Wood frog adaptations to overwintering in Alaska: new limits to freezing tolerance. The Journal of Experimental Biology 217: 2193-2200.

Layne, J., LeE, R. 1987. Freeze tolerance and the dynamics of ice formation in wood frogs (Rana sylvatica) from southern Ohio. Canadian Journal of Zoology 65: 2062-2065.

Layne, J., Lee, R. 1995. Adaptations of frogs to survive freezing. Climate Research 5: 53-59.

Layne, J., Lee, R., Cutwa, M. 1996 Post-hibernation excretion of glucose in urine of the freeze tolerant frog Rana sylvatica. Journal of Herpetology 30: 85-87.

Lobos, G., Vidal, M., Labra M., Correa, C., Rabanal, F., DíazPáez, H., Alzamora, A., Soto, C. 2012. Protocolo para el control de enfermedades infecciosas en Anfibios durante estudios de campo. URL. http://www.herpetologiadechile. cl/index.html. Accesado: Mayo 26, 2016.

Logares, R., Úbeda, C. 2006. First insights into the overwintering biology of Alsodes gargola frogs and tadpoles inhabiting harsh Andean-Patagonian alpine environments. AmphibiaReptilia 27: 263-267.

Luebert, F., Pliscoff, P. 2006. Sinopsis bioclimática y vegetacional de Chile. Primera edición, Editorial Universitaria, Santiago de Chile. 323 pp.

Navas, C. 1997. Thermal extremes at high elevations in the Andes: Physiological ecology of frogs. Journal of Thermal Biology 22: 467-477.

Navas, C., Úbeda, C., Logares, R., Jara, F. 2010. Thermal tolerances in tadpoles of three species of Patagonian anurans. South American Journal of Herpetology 5: 89-96.

Nova, R. 2010. Proyecciones sobre el estudio de la termofisiología de Calyptocephalella gayi en Chile y los impactos del cambio climático. Tesis de Grado ¿Magister, Doctorado? . Universidad del Biobío. Chillan. Chile.

Pinder, A., Storey, K., Ultsch, G. 1992. Estivation and hibernation. En: Feder, M., Burggren, W. (Eds). Environmental physiology of the amphybians: 250-274. The University of Chicago Press, Chicago.

Rosendale, A., Lee, R., Costanzo, J. 2014. Effect of Physiological Stress on Expression of Glucose Transporter 2 in Liver of 
the Wood Frog, Rana sylvatica. Journal of Experimental Zoology 321A: 566-576.

Sanabria, E., Quiroga, L., Acosta, J. 2003. Ecología térmica de Leptodactylus ocellatus (Linnaeus, 1758) (ANURA: Leptodactylidae) en los Banados de Zonda, San Juan, Argentina. Cuadernos de Herpetología 17: 127-129.

Sanabria, E., Quiroga, L., Acosta, J. 2007. Odontophrynus occidentalis $(\mathrm{NCN})$. Body temperature. Herpetological Review 38: 188-189.

Sanabria, E., Rodríguez, C., Vergara, C., Ontivero, E., Banchig, M., Navas, A., Herrera-Morata, M., Quiroga, L. 2015. Thermal ecology of the post-metamorphic Andean toad (Rhinella spinulosa) at elevation in the monte desert, Argentina. Journal of Thermal Biology 52: 52-57.

Sinclair, B., Addo-Bediako, A., Chown, S. 2003. Climatic variability and the evolution of insect freeze tolerance. Biological reviews 78: 181-195.

Sommaruga, R. 2001. The role of solar UV radiation in the ecology of alpine lakes. Journal of Photochemistry and Photobiology: Biology 62: 35-42.

Steiner, A., Petenusci, S., Brentegani, L., Branco, L. 2000. The importance of glucose for the freezing tolerance/ intolerance of the anuran amphibians Rana catesbeiana and Bufo paracnemis. Revista Brasileira de Biología 60: 321-328.
Storey, K., Storey, J. 1986. Freeze tolerant frogs: cryoprotectants and tissue metabolism during freeze-thaw cycles. Canadian Journal of Zoology 64: 49-54.

Storey, K., Storey, J. 1996. Natural freezing survival in animals. Annual Review of Ecology, Evolution, and Systematics 27: $365-386$

Storey, K., Storey, J. 2001. Hibernation: Poikilotherms. Encyclopedia of Life Sciences. Macmillan Publishers Ltd, Nature Publishing Group.

Tapley B., Acosta-Galvis A., Lopez J. 2011. A field method for sampling blood of male anurans with hypertrophied limbs. Phyllomedusa 10: 75-77.

Tattersall, G., Ultsch, G. 2008. Physiological Ecology of Aquatic Overwintering in Ranid Frogs. Biological Reviews 83: 119-140.

Victoriano P., Ortiz J.C., Troncoso L., Galleguillos R. 1995. Allozyme variation in populations of Pleurodema thaul (Lesson, 1826) (Anura; Leptodactylidae). Comparative Biochemistry and Physiology 112: 487-492.

Vidal, M., Labra A. 2008. Herpetología de Chile. Science Verlag Ediciones. Santiago de Chile. 594 pp.

Wells, K. 2007. The ecology and behavior of amphibians. The University of Chicago Press, Chicago and London. 1148 pp.

Recibido: 16.03 .2017

Aceptado: 20.10.2017 